\title{
Estabelecimento de valores de densidade mineral óssea (DMO) das regiões metafisária e diafisária do rádio em cães da raça Poodle por meio da Densitometria Óptica Radiográfica
}

Caterina MURAMOTO' ${ }^{1}$; Franklin de Almeida STERMAN ${ }^{1}$;

Ana Carolina Brandão de Campos FONSECA PINTO'

\section{Correspondência para:} FRANKLIN DE ALMEIDA STERMAN

Departamento de Cirurgia Faculdade de Medicina Veterinária e Zootecnia

Universidade de São Paulo Av. Prof. Orlando Marques de Paiva, 87 Cidade Universitária Armando Salles de Oliveira

05508-270 - São Paulo - SP

caterina@fmvz.usp.br

Recebido para publicação: 30/10/2003 Aprovado para publicação: 24/05/2004

1- Departamento de Cirurgia da Faculdade de Medicina Veterinária e Zootecnia da USP, São Paulo - SP

\section{Resumo}

A Densitometria Óptica Radiográfica permite aferir o conteúdo mineral do osso por meio de imagens radiográficas, contribuindo para o diagnóstico de osteopenia, monitoração seriada da densidade em resposta a enfermidades ósseas, a intervenções cirúrgicas e de terapia, e a estratégias preventivas relacionadas ao metabolismo ósseo. As densidades ópticas foram medidas por meio do software de processamento de imagens ImageLab, cuja aplicabilidade foi testada para determinação de valores de referência de DMO, comparandoos a uma escala padrão confeccionada em alumínio, radiografada junto ao osso. Correlacionou-se os valores de DMO com as variáveis peso, idade, sexo e largura e espessura do terço distal do rádio-ulna direito de 112 cães Poodle. Pela diferença na proporção de osso esponjoso e compacto entre as regiões metafisária e diafisária, os animais apresentaram a DMO da metáfise menor que a da diáfise, fato não observado no grupo de animais de até um ano de idade, devido ao alto metabolismo ósseo na região metafisária durante o período de crescimento. A análise dos valores médios de DMO obtidos para cada categoria avaliada, permitiu verificar que a classificação mais adequada para estabelecer valores de referência foi a por peso e região óssea. Assim, foram estabelecidos valores médios para a região metafisária (M) e para as diafisárias 1 (D1) e 2 (D2) em três categorias de peso. Para cães de até $2,5 \mathrm{~kg}$ os valores definidos foram: DMO-M=2,55 $\pm 0,30$, DMO-D1 $=2,89 \pm 0,40 \mathrm{e}$ DMO-D2 $=3,16 \pm 0,43 \mathrm{mmAl}$; para os de 2,6 a 5,0kg foram: DMO$\mathrm{M}=3,16 \pm 0,43, \mathrm{DMO}-\mathrm{D} 1=3,67 \pm 0,47$ e DMO-D2 $=3,96 \pm 0,55 \mathrm{mmAl}$ e para os de 5,1 a $7,5 \mathrm{~kg}$ foram: DMO-M=3,72 $\pm 0,50$, DMO$\mathrm{D} 1=4,31 \pm 0,52$ e DMO-D2=4,60 $\pm 0,62 \mathrm{mmAl}$.
Palavras-chave:

Densidade mineral óssea.

Densitometria óptica radiográfica. Radiologia.

Cães.

\section{Introdução}

Muitos fatores ligados ao metabolismo ósseo sejam externos, internos, sistêmicos ou locais exercem influência sobre o processo de remodelação óssea e muitas lacunas ainda precisam ser preenchidas em relação à explicação deste processo em que, estresse mecânico, nutrição, atuação de vitamina D, calcitonina, hormônio paratireóideo (PTH) e hormônios sexuais exercem importante papel ${ }^{1,2}$. Mundy ${ }^{3}$ descreve vários fatores que, direta ou indiretamente, atuam na ativação ou inibição da remodelação óssea. Dentre os que levam à ativação de osteoclastos, estimulando a reabsorção óssea estão o PTH, 1,25diidroxivitaminaD 3, interleucina 1, linfotoxina, fator de necrose tumoral, fator estimulador de colônia 1, interleucina 6, vitamina $A$, leucotrienos, hormônios tireoidianos e glicocorticóides. Em relação 
aos inibidores da reabsorção óssea osteoclástica são citados calcitonina, interleucina 8, ODIR/RANK ligand, interferon $\mathrm{g}$, fator de crescimento transformador-b e alguns agentes farmacológicos como nitrato de Gallium e bifosfonatos.

A avaliação do status do esqueleto pode ser feita por meio de medidas de densidade mineral óssea (DMO) as quais podem auxiliar na instituição do diagnóstico de osteopenia, na monitoração seriada do osso em resposta a patologias, a intervenções cirúrgicas e terapêuticas e em estratégias preventivas relacionadas ao metabolismo ósseo ${ }^{4}$.

A densitometria óptica radiográfica mede a DMO por meio de imagens radiográficas, apresentando grande importância na área de pesquisa e clínica pela capacidade em auxiliar o profissional de saúde a compreender e avaliar o processo de mineralização óssea com boa precisão e sensibilidade, reprodutibilidade, fácil aplicabilidade e baixo custo, salientando-se que o tempo de execução é pequeno e não necessita nenhum procedimento anestésico. Todas essas características estimulam o emprego da metodologia já que, tendo em vista a atual condição sócio-econômica da maioria da população brasileira, o custo do método a ser utilizado é socialmente relevante.

Sabe-se, porém, que a técnica radiográfica está sujeita a um grande número de variáveis que dificultam ou impedem sua padronização, destacando-se a influência da quilovoltagem, da miliamperagem, do efeito anódico e do tempo de exposição ${ }^{5}$. Uma das maneiras de contornar estes problemas é o estabelecimento de curvas de calibração provenientes dos valores de densitometria óptica radiográfica de um penetrômetro, uma escala graduada de características conhecidas, de maneira a servir como um padrão referencial densitométrico. Assim, pode-se converter os valores de densidade óptica radiográfica para valores em espessura de um padrão radiografado simultaneamente à região a ser analisada, sendo que, muitos trabalhos adotaram a conversão para valores em milímetros de alumínio ${ }^{6}$. A escolha do alumínio para confecção da escala de referência se deve a este material exibir uma curva de absorção da radiação X semelhante à dos $\operatorname{Ossos}^{7,8}$.

A imagem da escala de alumínio obtida no filme radiográfico reflete as condições de processamento e a exposição a que o filme foi submetido e a curva de densidade produz dados necessários para corrigir a análise do osso por meio de integração, gerando uma forma de determinação comparativa da absorção dos raios $\mathrm{X}^{7}$.

O processamento de imagens permite realização de alterações e análise das informações relativas a elas, produzindo uma melhora na qualidade da imagem utilizada pelo observador humano, o qual é capaz de distinguir de 28 a 32 diferentes tons de cinza em uma radiografia convencional, o que é muito inferior ao potencial de informações representado pelo ambiente digital do computador, que permite ajustes de contraste de até 256 tons de cinza ${ }^{9}$. Segundo Jeffcoat ${ }^{10}$, devido às limitações visuais, para a osteoporose ser detectada radiologicamente é necessário que esteja em uma fase avançada com pelo menos 30 a 50\% de perda de cálcio do osso.

Os cães da raça Poodle, por serem bastante comuns em nosso meio, permitenos supor que, com uma freqüência relativamente grande, estarão entre os animais acometidos por diversas enfermidades que podem influenciar no metabolismo ósseo. Seria difícil agrupar cães de diferentes raças em relação a categorias que possuam semelhança nos valores de DMO, pois são, provavelmente, os mamíferos mais polimorfos referidos como uma única espécie. Riser ${ }^{11}$ chega a mencionar que até 2000 raças já foram desenvolvidas no mundo, sendo que nos Estados Unidos o AKC reconhece 148 raças, segundo a publicação oficial de 1998.

Sendo assim, este trabalho propõe a 
avaliação dos valores de densidade mineral óssea em cães de uma mesma raça, obtidos através da técnica de densitometria óptica radiográfica, utilizando software de processamento de imagem ImageLab, com a finalidade de verificar a viabilidade da metodologia empregada, correlacionar a densidade mineral óssea com as variáveis estudadas (peso, idade, sexo, largura e espessura do terço distal do rádio-ulna) e de estabelecer os valores de referência da densidade mineral óssea em equivalentes a milímetros de alumínio para a raça em questão.

Objetivou-se também, correlacionar os valores das leituras de densidade óssea da região diafisária e da metafisária do rádio no mesmo animal e verificar se a razão entre o comprimento crown-rump e a altura dos animais pode ser considerada como valor de referência para estabelecimento de um tipo constitucional.

Com o estabelecimento de valores normais da densidade mineral óssea e com a verificação de que a metodologia utilizada é apropriada para tal avaliação, poderão ser realizadas pesquisas com cães que possuam alterações na mineralização óssea decorrentes de diversas condições patológicas e/ou fisiológicas.

\section{Materiais e Métodos}

Neste experimento foram utilizados 112 cães da raça Poodle, sendo 59 fêmeas e 53 machos, com idades variando entre 3 e 168 meses (14 anos) e peso variando entre 1,1 e 7,5 quilogramas (kg), passados por uma avaliação prévia, a fim de selecionar apenas cães sem alterações ósseas evidentes.

Para cada animal foi preenchida uma ficha individual com a identificação do animal e com os valores das medidas das variáveis coletadas (largura e espessura da região correspondente ao terço distal do rádio e ulna direitos, peso, altura e comprimento crown-rump).

Animais ovariectomizados ou orquiectomizados e aqueles acima de $7,5 \mathrm{Kg}$ foram descartados deste estudo.

O grupo total de animais foi dividido em 6 subgrupos, assim caracterizados: G1 (10 fêmeas com até um ano de idade); G2 (33 fêmeas com idade variando entre 1 e 7 anos); G3 (16 fêmeas com mais de 7 anos de idade); G4 (11 machos com até um ano de idade); G5 (20 machos com idade variando entre 1 e 7 anos) e G6 (22 machos com mais de 7 anos de idade).

Foram selecionados 60 animais a partir do grupo total de 112 animais, com o objetivo de constituir um grupo mais homogêneo em relação à categoria peso, denominado grupo selecionado. Para a formação deste grupo, foram escolhidos 10 animais, de cada um dos seis subgrupos (G1 a G6), com o critério de semelhança de pesos corpóreos. Estes seis novos grupos constituintes do grupo selecionado foram denominados GS1, GS2, GS3, GS4, GS5 e GS6.

Todas as radiografias foram feitas com chassi metálico marca Kodak Lanex equipado com tela intensificadora "regular screens" e filme marca Kodak MXG/Plus de $18 \mathrm{x} 24 \mathrm{~cm}$, em aparelho de raio X de marca Ray tec, modelo RT 500/125, com ampola Toshiba Rotanode Tm Fluray unit model E 7239 X. A técnica radiográfica utilizada para a obtenção de todas as radiografias foi fixada em 40 quilovolts $(\mathrm{kV}), 100$ miliampères $(\mathrm{mA}), 0,016$ segundos (s) e a distância entre o foco e o filme de 1 metro.

Foi desenvolvida uma escala de referência densitométrica, confeccionada em alumínio (liga padronizada pela ABNT) contendo 20 degraus, sendo o primeiro de 0,5 milímetro $(\mathrm{mm})$ de altura e os demais aumentando de $0,5 \mathrm{em} 0,5 \mathrm{~mm}$.

Primeiramente, foi feita uma radiografia apenas da escala de alumínio, cuja imagem radiográfica foi denominada de imagem padrão e após ser escaneada em Scanner Hewlett Packard (HP ScanJet 6300C, com adaptador de transparência HP ScanJet XPA), foi armazenada no Microcomputador (Pentium III Processor de 450MHz; 128,0Mb de memória RAM e Winchester de 12Gb de 
memória), como referência para comparação posterior com a imagem da mesma escala de alumínio, radiografada simultaneamente ao osso cuja densidade óptica seria medida. $\mathrm{O}$ rádio direito de cada animal foi então radiografado juntamente com a escala de alumínio, a qual foi posicionada medial e paralelamente ao eixo longitudinal do rádio, com o auxílio de um porta chassi, confeccionado em madeira.Após terem sido reveladas em processadora automática (Processadora automática RP-X-OMAT/ Kodak Eastman Company), as radiografias foram escaneadas e salvas na cor escala de cinzas, no modo bmp (bitmap) e armazenadas em compact disk (CD).

Para realização das leituras das densidades ópticas (DO) referentes a DMO em milímetros de alumínio ( $\mathrm{mmAl}$ ) foi utilizado o software de processamento e análise de imagem ImageLab, desenvolvido pela empresa Softium Sistema de Informática Ltda. Me.

$\mathrm{Na}$ imagem padrão, selecionou-se os dezenove primeiros degraus da escala de alumínio e obteve-se uma planilha contendo os valores de DO de cada degrau da imagem padrão que variam de 1 a 19 conforme o degrau da escala. Lateralmente à planilha é representada uma curva densitométrica em um gráfico cujo eixo $\mathrm{x}$ representa as densidades ópticas (de 1 a 19) e o eixo y representa os tons de cinza, que variam neste programa, de zero a 256 tons. Pressionandose o botão "Padronizar", o programa fixa os valores determinados para a imagem padrão e, toda imagem da escala dos arquivos abertos posteriormente, em seqüência, pode ser comparada a este padrão.

Foi utilizado um artifício do programa ImageLab que equaliza imagens para corrigir possíveis diferenças nos tons de cinza, decorrentes das variações que a imagem radiográfica pode sofrer durante o processamento. Para isso, na imagem radiográfica da escala de alumínio radiografada simultaneamente ao osso, foram selecionados os mesmos dezenove degraus (Figura 1) e obtida uma nova planilha e um gráfico contendo a curva densitométrica da imagem padrão, em vermelho, e a curva densitométrica da nova imagem selecionada, em azul (Figura 2). Como se trata da mesma escala de alumínio, radiografada com a mesma técnica, apenas em tempo e filmes radiográficos diferentes, foi pressionado o botão "Equalizar", ordenando ao programa considerar as duas imagens como sendo iguais. Desta forma, o programa capta, por subtração de imagem, as diferenças entre as duas imagens e as aproxima da imagem padrão. A mesma aproximação que o programa utiliza para equalizar os tons da escala de alumínio é utilizada, por ele, para modificar todos os tons de cinza do arquivo aberto, ou seja, altera, da mesma maneira, os tons de cinza da imagem radiográfica do osso.

Foram demarcadas 3 regiões na imagem do osso rádio: uma na região metafisária e duas na região diafisária.

O tamanho da área a ser selecionada de cada região foi determinado por um quadrilátero com os dois lados paralelos correspondendo a largura do rádio e com a altura correspondendo a metade do comprimento da largura. No momento de selecionar o comprimento da largura, incluiuse uma extensão apenas de partes moles, com a finalidade de poder subtrair a densidade das partes moles sobrepostas ao osso já que, para determinação da DO, será feita uma leitura média dos tons de cinza de toda a área selecionada.

A base do quadrilátero delimitado na região metafisária é posicionada na região imediatamente dorsal ao disco ou cicatriz epifisária. A primeira região diafisária demarcada (D1) inicia-se um centímetro após o limite da área demarcada para a região metafisária e a região diafisária 2 (D2), um centímetro após o limite da região D1.

Selecionadas as regiões, pressiona-se o ícone cálculo de regiões, que gera os valores de densidade óptica para cada uma delas (Figura 3). O valor de densidade óptica gerado é dado em milímetros de alumínio, uma vez que o tom de cinza obtido na região selecionada do osso será comparado aos tons 


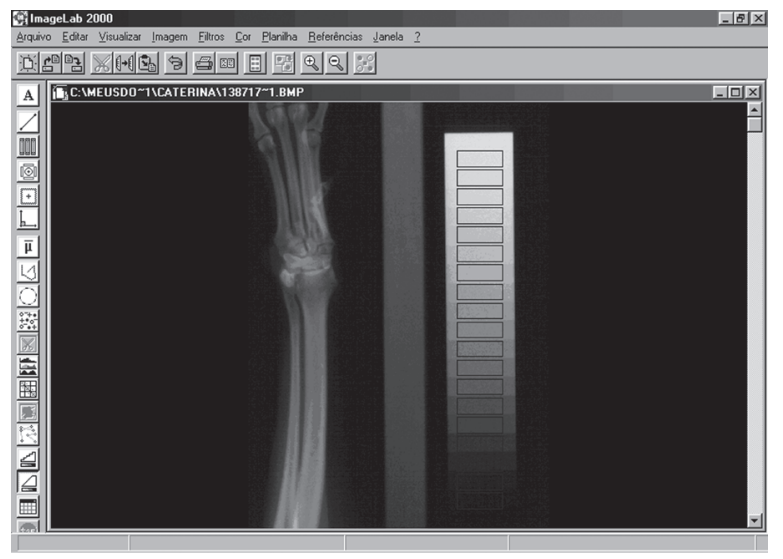

Figura 1 - Visualização do programa ImageLab mostrando o rádio-ulna direito posicionado paralelamente a escala de referência de alumínio, com 19 degraus selecionados

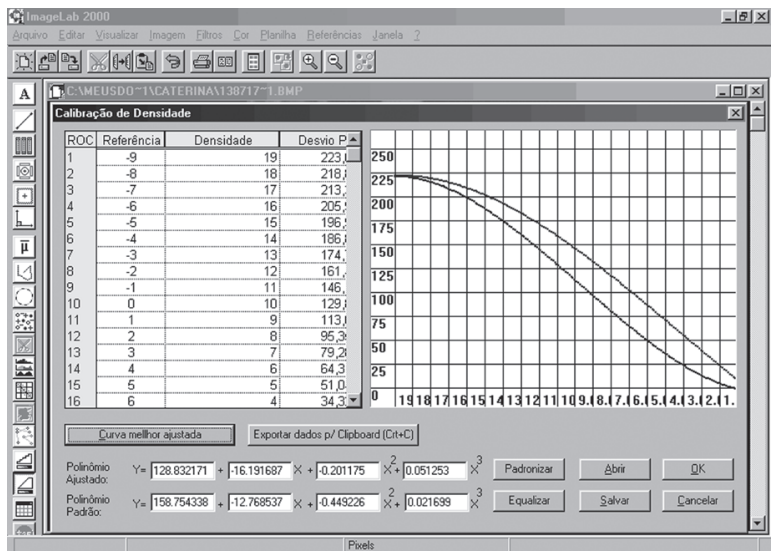

Figura 2 - Visualização da planilha e do gráfico que mostra uma curva (em vermelho) representativa dos valores da densidade óptica dos degraus da imagem padrão da escala de alumínio e uma segunda curva (em azul) representativa dos degraus da escala de alumínio radiografada simultaneamente ao osso antes da equalização

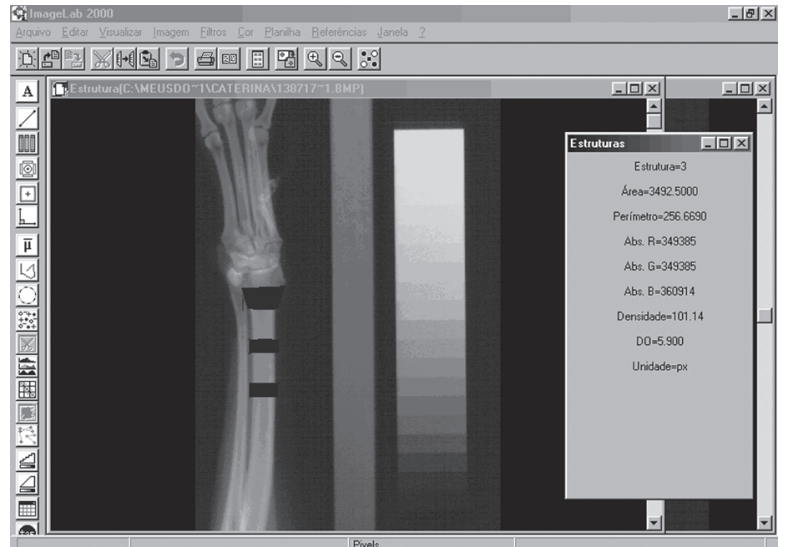

Figura 3 - Imagem do programa ImageLab mostrando os três quadriláteros correspondentes as regiões metáfisária (M), diafisária 1 (D1) e diafisária 2 (D2) demarcadas e a leitura da densidade óptica (DO) da região metafisária 
dos degraus da escala de referência confeccionada em alumínio, já equalizada com a imagem padrão.

O procedimento de demarcação e de leitura das DO das 3 regiões foi repetido por mais duas vezes, a fim de se calcular uma média de três leituras para cada animal radiografado.

Obtida a média das três leituras de cada região, cada um dos valores médios foi dividido por 2, para se obter o valor da densidade mineral óssea (DMO) em mmAl, uma vez que cada degrau possui $0,5 \mathrm{~mm}$ de altura.

\section{Resultados e Discussão}

Este trabalho permitiu comprovar que a Densitometria Óptica Radiográfica é uma metodologia de fácil aplicação, baixo custo, segura e de boa precisão, em concordância com autores de outros estudos, que utilizaram a mesma metodologia ${ }^{12,13,14}$.

Os animais foram submetidos a uma técnica radiográfica convencional, com baixo índice de radiação, não necessitando qualquer tipo de anestesia ou sedação para efetuar o posicionamento do membro do animal sobre o chassi, o qual foi obtido e mantido apenas por poucos segundos com o auxílio do proprietário.

Dentre as variáveis estudadas no presente trabalho, as duas mais importantes para caracterizar um determinado cão dentro de um ou outro grupo de referência foram: a razão crown-rump por altura (CR/h), a qual dá uma boa idéia de proporção do animal, e o peso, ambas analisadas conjuntamente.

Neste trabalho, assim como era esperado, por se tratarem de cães da mesma raça, apenas de portes diferentes, a razão CR/

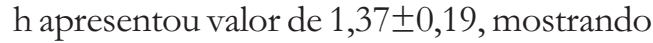
um desvio-padrão mínimo, coerente com o fato de a proporção entre comprimento e altura manter-se praticamente iguais em animais de mesmo tipo constitucional.

A média de DMO-média (média entre as 3 regiões: M, D1 e D2) encontrada foi de $3,39 \pm 0,63 \mathrm{mmAl}$ para as fêmeas e de $3,69 \pm 0,72 \mathrm{mmAl}$ para os machos com valor de $3,53 \pm 0,69 \mathrm{mmAl}$ para o grupo total. Em relação às categorias de peso, foram encontrados valores médios de DMO-média de $2,86 \pm 0,34 \mathrm{mmAl}$ para animais de até $2,5 \mathrm{~kg}$, de $3,60 \pm 0,45 \mathrm{mmAl}$ para os cães de 2,6 a $5,0 \mathrm{~kg}$ e de $4,21 \pm 0,53 \mathrm{mmAl}$ para os de $5,1 \mathrm{a} 7,5 \mathrm{~kg}$. Em se tratando de categorias por idade, os valores encontrados foram de $3,20 \pm 0,67 \mathrm{mmAl}$ para animais de até 1 ano de idade, de $3,52 \pm 0,66 \mathrm{mmAl}$ para os de idade variando entre 1 e 7 anos e de $3,73 \pm 0,67 \mathrm{mmAl}$ para aqueles com mais de 7 anos de idade.

A análise dos valores médios de DMOmédia obtidos para cada variável em suas diferentes categorias, permitiu verificar que o peso foi a que melhor conseguiu definir os valores de referência com a finalidade de classificação do animal a ser estudado, apresentando maior correlação com a DMO, de modo que a equação de variação de DMOmédia em função do peso conseguiu explicar $61 \%$ (coeficiente de determinação r) da variação da DMO-média. A forte correlação entre peso e DMO foi também evidenciada por outros autores tanto em humanos como em animais ${ }^{15,16,17}$. Adicionando-se outras variáveis a esta função, por meio de uma análise de regressão múltipla, verificou-se que, ao introduzir a variável idade, o coeficiente de determinação aumentou muito discretamente, atingindo o valor de $61,6 \%$, índice justificado pela fraquíssima correlação entre idade e DMO-média obtida neste estudo. Introduzindo-se a variável largura, nesta função de regressão múltipla, o coeficiente foi para $63 \%$ e, finalmente, ao adicionar a espessura, o coeficiente chegou a $64,9 \%$, indicando, de qualquer modo, que ainda restam $35,1 \%$ dos valores de DMO-média que não são explicados pela função DMO-média por peso, idade, largura e espessura, e que, portanto, há outras variáveis, não analisadas no presente estudo, exercendo influência considerável sobre a variação da $\mathrm{DMO}$.

Analisando-se o coeficiente de determinação da DMO-média com a idade, com a largura e com a espessura, isoladamente, foram obtidos resultados de $6 \%, 37 \%$ e $43 \%$, respectivamente, indicando 
que as retas de regressão linear não conseguem explicar nem a metade da variação da DMO. Por isso, de acordo com Costa $\mathrm{Neto}^{18}$, não se deve nem cogitar em estabelecer funções de variação de DMO por uma destas três variáveis.

Em relação a idade, não foi encontrada diferença significativa entre os grupos de animais de 1 a 7 anos e o grupo de animais acima de 7 anos. Já os valores de DMO-média do grupo de animais de 1 a 7 anos foram significativamente maiores que os do grupo de até um ano de idade, mas como a média de peso dos mais jovens foi menor que a dos animais adultos, foi indagado se a diferença de densidade não seria apenas por influência do peso. Para esclarecer esta dúvida, foi analisado o resultado para o grupo de animais selecionados, verificando-se não haver diferença estatisticamente significante entre os grupos de faixas etárias diferentes.

Apesar da DMO-média dos animais do sexo masculino ser, significativamente, maior do que a do sexo feminino no grupo total de 112 animais, o peso médio do grupo de machos também foi maior que o de fêmeas, surgindo, novamente a indagação se não seria influência da variável peso, já que este, exerce forte correlação com a DMO. Constatou-se, pela aplicação do Teste-t de Student nos animais do grupo selecionado, que, entre os 30 machos e as 30 fêmeas com pesos semelhantes, não houve diferença significativa entre as DMO, concluindo-se que o peso exerceu influência para a diferença encontrada no grupo total.

Analisando os valores de DMO do grupo total de 112 animais para as diferentes regiões do osso, verificou-se haver diferença significativa entre a DMO-M e DMO-D1 e entre a DMO-D1 e DMO-D2. A porcentagem de diferença nos valores de DMO entre a região metafisária e diafisária do grupo total $(15 \%)$ foi bem maior que a porcentagem entre as regiões diafisárias (8\%), fato justificado pela maior proporção de osso esponjoso em relação ao osso compacto na região metafisária e maior quantidade de osso compacto na diáfise, o qual vai tornando-se mais espesso em direção ao terço médio dos ossos longos ${ }^{1}$.

Apenas os grupos de animais com menos de 1 ano, por apresentarem alto metabolismo ósseo na região metafisária, com taxa de deposição óssea maior que a taxa de absorção ${ }^{15}$, não tiveram diferença significativa entre as densidades das diferentes regiões do osso. Todas as outras categorias de idade, em ambos os sexos, apresentaram DMO-M significativamente menor que DMO-D1. Em relação às duas regiões diafisárias, por se distanciarem apenas em 1 centímetro, não foram encontradas diferenças estatisticamente significantes, provavelmente pela inexistência de grande variação da espessura do osso compacto.

Finalizando, pode-se afirmar que essa metodologia tem aplicação pertinente para pesquisas que envolvam metabolismo ósseo e para estudo e acompanhamento de patologias e terapias que necessitem da avaliação da DMO como ferramenta direcionadora de condutas.

No campo da Medicina Veterinária há carência de estudos definidores dos valores de referência de DMO para animais de diferentes espécies e raças. Para os animais, para os quais não existam valores de referência definidos, essa metodologia pode ser utilizada efetuando-se medições cronologicamente seriadas para aferir a evolução de um determinado quadro.

\section{Conclusões}

A utilização do software de processamento de imagens ImageLab, mostrou-o como sendo de fácil manuseio e apresentando boa precisão para a leitura das densidades ópticas das imagens radiográficas do rádio de cães.

Os valores de referência de DMO para cães da raça Poodle determinados por este trabalho, foram estabelecidos levando em consideração a variável peso e a região óssea. Desta forma, para os animais com até $2,5 \mathrm{~kg}$ de peso corporal os valores foram: 
DMO-M=2,55 $\pm 0,30 ;$ DMO-D1 $=, 88 \pm 0,40$ e DMO-D2 $=3,16 \pm 0,43$. Para os com peso variando entre 2,6 a $5,0 \mathrm{~kg}$ foram: DMO$\mathrm{M}=3,16 \pm 0,43$; DMO-D1=3,67 $\pm 0,47 \mathrm{e}$
DMO-D2 $=3,96 \pm 0,55$. Para os animais entre 5,1 e $7,5 \mathrm{~kg}$ os valores de referência foram: DMO-M=3,72 $\pm 0,50 ;$ DMO-D1 $=4,41 \pm 0,52$ e DMO-D2=4,60 $\pm 0,62$.

\title{
Establishment of values of bone mineral density (BMD) from radius's metaphysary and diaphysary regions in Poodle by Radiographic Optic Densitometry
}

\begin{abstract}
Radiographic Optic Densitometry was used to measure bone mineral content by radiographic images. This method contributes to the diagnosis of osteopenia, preventive estrategies associated to bone metabolism and monitoring of series of measurement of density to accompany pathologies, surgeries, therapies and. The optic density was measured by the image processing software, ImageLab; the applicability of this method was tested and canine reference values of bone mineral density (BMD) were compared to a standard aluminium scale radiographed with the bone. Correlations between the BMD values and the variables weigth, age, sex and width and thickness of the distal third of the 112 Poodles's right radius-ulna were studied. It was observed that diaphysary BMD values were higher than metaphysary BMD values due to the differences between cancelous and compact bones. However, these differences in the $\mathrm{BMD}$ values were not observed in the younger group, up to about one year of age, probably due to the higher bone metabolism during the growing period in the metaphysary region. The average BMD values for each weight category showed that the best method to establish reference values was by weight and bone region. Average values of metaphysary region (M) and diaphysaries 1 (D1) and 2 (D2) were established in three different weight categories. For dogs up to $2.5 \mathrm{~kg}$ in weight these values were $\mathrm{BMD}-\mathrm{M}=2.55 \pm 0.30$, BMD$\mathrm{D} 1=2.89 \pm 0.40$ and $\mathrm{BMD}-\mathrm{D} 2=3.16 \pm 0.43 \mathrm{mmAl}$; for dogs from 2.6 up to $5.0 \mathrm{~kg}$ these values were $\mathrm{BMD}-\mathrm{M}=3.16 \pm 0.43$, BMD$\mathrm{D} 1=3.67 \pm 0.47$ and BMD-D2 $=3.96 \pm 0.55 \mathrm{mmAl}$ and for dogs from 5.1 up to $7.5 \mathrm{~kg}$ these values were $\mathrm{BMD}-\mathrm{M}=3.72 \pm 0.50$, BMD$\mathrm{D} 1=4.31 \pm 0.52$ and BMD-D2 $=4.60 \pm 0.62 \mathrm{mmAl}$.
\end{abstract}

\section{Referências}

1 EVANS, H. E. The Skeleton. In: EVANS, H. E. Miller's anatomy of the dog. Philadelphia: WB Saunders, 1993. p.122-191.

2 WASSERMAN, R.H.; KALLFELZ, F. A.; LUST, G. Ossos, articulações e líquido sinovial. In: REECE, W. O.; SWENSON, M. J. Dukes/fisiologia dos animais domésticos. Rio de Janeiro: Guanabara Koogan, 1996. p.488-520.

3 MUNDY, G. R. Bone remodeling. In: FAVUS, M. J. Primer on the metabolic bone diseases and disorders of mineral metabolism. Philadelphia: Lippincott Williams
Key-words:

Bone mineral density. Radiographic optic densitometry. Radiology.

Dogs
\& Wilkins, 1999. p. 30-38.

4 MILLER, P. D.; BONNICK, S. L. Clinical application of bone densitometry. In: FAVUS, M. J. Primer on the metabolic bone diseases and disorders of mineral metabolism. Philadelphia: Lippincott Williams \& Wilkins, 1999. p. 152-159.

5 VOGEL, J. M.; ANDERSON, J. T. Rectilinear transmission scanning of irregular bones for quantification of mineral content. Journal of nuclear medicine, v.13, n.1, p.13-18, 1971.

6 LOUZADA, M. J. Q. Otimização da técnica de 
densitometria óptica em imagens radiográficas de peças ósseas. Estudo "in vitro". 1994. Tese (Doutorado em engenharia elétrica) - Faculdade de Engenharia Elétrica, UNICAMP, Campinas, 1994.

7 MACK, P. B.; VOSE, G. P.; NELSON, J. D. New development in equipament for the roentgenographic measurement of bone density. American Journal of Roentgenology, v. 82, n. 2, p. 303-310, 1959.

8 OWEN, M. Measurement of the variations in calcification in normal rabbit bone. The Journal of Bone and Joint Surgery, v. 38B, n. 3, p. 762-769, 1956.

9 BAXES, G. A. Digital image processing - principles and applications. New York: John Wiley \& Sons, 1994. $452 \mathrm{p}$.

10 JEFFCOAT, M. K. Radiographic Methods for the Detection of Progressive Alveolar Bone Loss. Jounal of Periodontology, n. 63, p. 367-372, 1992.

11 RISER, W. H. Carnívoro - Introdução. In: GETTY, R. Sisson/Grossman anatomia dos animais domésticos. Rio de Janeiro: Guanabara Koogan, 1986. v. 2, p. 1335-1336.

12 LOUZADA, M. J. Q.; PELÁ, C. A.; BELANGERO, W. D.; SANTOS-PINTO, R. Avaliações de densidade óssea em imagens radiográficas: estudo em peças ósseas de cães. RBE - Caderno de Engenharia Biomédica, v. 14, n. 1, p. 47-64, 1998.

13 STERMAN, F. A. Avaliação da densidade óssea em eqüinos atletas destinados ao Enduro Eqüestre. 2002. Tese (Livre Docência) - Faculdade de Medicina Veterinária e Zootecnia - Universidade de São Paulo, São Paulo, 2002.

14 VULCANO, L. C.; CIARLINI, L. D. R. P.; LOUZADA, M. J. Q.; CALDAS, E. L. C. Valores normais da densidade óssea do carpo ulnar em potros em crescimento da raça Quarto de Milha através da densitometria óptica radiográfica. A Hora Veterinária. v. 17, n. 100, 1997.

15 GUYTON, A. C.; HALL, J. E. Tratado de Fisiologia Médica. Rio de Janeiro: Guanabara Koogan, 1997. p. 895-910.

16 STOLIKER, H. E.; DUNLAP, H. L.; KRONFELD, D. S. Bone mineral measurement by photon densitometry in racing sled dogs and its relationship to body weight, sex, and bone fractures. Veterinary Medicine. Small Animal Clinician, november, p. 1545-1550, 1976.

17 TALBOTT, S. M.; CIFUENTES, M.; DUNN, M. G.; SHAPSES, S. A. Energy restriction reduces bone density and biomechanical properties in aged female rats. Journal of Nutrition, n. 131, p. 2382-2387, 2001.

18 COSTA NETO, P. L. O. Estatística. São Paulo: Edgard Blücher, 1995. p. 178-228. 